\title{
Environmental studies in Lake Baikal: basic facts and perspectives for interdisciplinary research
}

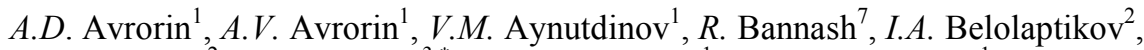 \\ V.B. Brudanin ${ }^{2}$, N.M. Budnev ${ }^{3, *}$, A.A. Doroshenko ${ }^{1}$, G.V. Domogatsky ${ }^{1}$, R. Dvornický ${ }^{2,8}$, \\ A.N. Dyachok ${ }^{3}, Z$ Zh.-A.M. Dzhilkibaev ${ }^{1}, L$. Fajt ${ }^{2,8,9}, S . V$. Fialkovsky ${ }^{5}, A . R$. Gafarov $^{3}$, \\ K.V. Golubkov ${ }^{1}$, N.S. Gorshkov ${ }^{2}$, T.I. Gres ${ }^{3}, K . G . \mathrm{Kebkal}^{7}, O . G . \mathrm{Kebkal}^{7}$, E.V. Khramov',

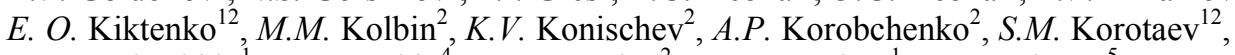

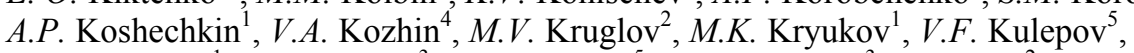

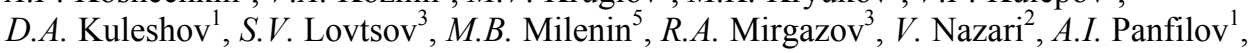 \\ D.P. Petukhov ${ }^{1}$, E.N. Pliskovsky ${ }^{2}$, I.A. Portyanskaya ${ }^{3}$, M.I. Rozanov ${ }^{6}, E . V$. Rjabov $^{3}$, \\ V.D. Rushay ${ }^{2}$, G.B. Safronov ${ }^{2}$, V.O. Serdyuk ${ }^{12}$, B.A. Shaibonov ${ }^{2}, F$. Simkovic ${ }^{2,8}$, \\ M.D. Shelepov ${ }^{1}, I$. Shtekl $^{9}, A . G$. Solovjev ${ }^{2}, M . N$. Sorokovikov ${ }^{2}, M$. Sturm $^{10}$, \\ O.V. Suvorova ${ }^{1}$, V.A. Tabolenko ${ }^{3}$, B.A. Tarashansky ${ }^{3}$, E.S. Troitskaya ${ }^{11}$, S.A. Yakovlev ${ }^{7}$, \\ and $A . V$. Zagorodnikov ${ }^{3}$ \\ ${ }^{1}$ Institute for Nuclear Research, Moscow, 117312 Russia \\ 2 Joint Institute for Nuclear Research, Dubna, 141980 Russia \\ ${ }^{3}$ Irkutsk State University, Irkutsk, 664003 Russia \\ ${ }^{4}$ Institute of Nuclear Physics, Moscow State University, Moscow, 119991 Russia \\ ${ }^{5}$ Nizhni Novgorod State Technical University, Nizhni Novgorod, 603950 Russia \\ ${ }^{6}$ St. Petersburg State Marine Technical University, St. Petersburg, 190008 Russia \\ ${ }^{7}$ EvoLogics, Berlin, Germany \\ ${ }^{8}$ Comenius University, Bratislava, Slovakia \\ ${ }^{9}$ Czech Technical University in Prague, Prague, Czech Republic \\ ${ }^{10}$ Swiss Federal Institute of Aquatic Science and Technology (Eawag), Kastanienbaum, Switzerland \\ ${ }^{11}$ Limnological Institute, Irkutsk 664033, Russia \\ ${ }^{12}$ Schmidt Institute of Physics of the Earth, Moscow 142190 Russia
}

\begin{abstract}
Lake Baikal in Siberia is one of the most interesting lakes in the world. It is the world's largest reservoir of fresh surface water and home to several hundred endemic species. At the same time it harboured the first underwater neutrino telescope NT200, now followed by its successor Baikal-GVD, a cubic-kilometre scale neutrino telescope. Within the Baikal Neutrino project a number of methods and instruments have been designed to study various processes in the Baikal ecosystem. Hundreds of optical, acoustic and other sensors allow for long-term 3D monitoring of water parameters like temperature, inherent optical properties or the intensity of water luminescence, as well as processes like sedimentation or deep water renewal. Here we present selected results of the interdisciplinary environmental studies.
\end{abstract}

* Corresponding author: nbudnev@api.isu.ru 


\section{Introduction}

Lake Baikal, the world's oldest, deepest and most voluminous fresh-water lake, is one of the most important biodiversity hotspots on the planet, holding about $20 \%$ of the world's total freshwater. It is located in eastern Siberia covering an area of $31,722 \mathrm{~km}^{2}$. Being an isolated-rift lake for 25 million years it contains an intriguing sedimentary record for palaeoclimate reconstructions. Moreover it is home to more than 2000 endemic species populating the lake's entire water volume. It also harboured the first underwater Neutrino Telescope NT200, deployed 1993-1998, and presently its successor, the Gigaton Volume Detector Baikal-GVD [1].

The progress of the Baikal Neutrino Experiment is connected, to a considerable degree, to the favourable natural conditions of the site. Naturally, site studies are a significant part of the project -- not only because the configuration of neutrino detectors builds on the knowledge of light background, optical characteristics of water, water currents and other properties, but also because new technologies, instruments and methods designed in the framework of the experiment allow to get much information about the Lake Baikal ecosystem. First of all, continuous, long-term monitoring of water parameters (temperature, bio- and chemo-luminescence, water currents etc.) with a many different instruments distributed on the moorings of the neutrino observatory allows studying horizontal and vertical water exchanges which are of great importance for the preservation of the Baikal ecosystem. The water currents supply all layers of the $1640 \mathrm{~m}$ deep lake with oxygen, redistribute solved and suspended matter and prevent accumulation of toxic substances. Dependent on specific geographical, meteorological and other factors, a number of different hydrophysical phenomena take place: flows, fronts, internal waves, upwelling, convection, turbulence eddies an so on, with space-time scales ranging from a fraction of millimetres and seconds up to several hundreds of kilometres and several years.

\section{Luminescence of Baikal water}

We started the study of the natural light background in Lake Baikal in 1981, with first investigations of the depth dependence of sunlight. Below $500 \mathrm{~m}$ depth, the sun's light field is less than that from water luminescence, below $800 \mathrm{~m}$ it is less than 1 photon $/\left(\mathrm{cm}^{2} \times \mathrm{sec}\right)$. Also the luminescence decreases with depth (see Fig.1), and at $1000 \mathrm{~m}$ below surface its photon flux density amounts to a few hundred photons $/\left(\mathrm{cm}^{2} \times \mathrm{sec}\right)$. With many different experiments we found that this luminescence is primarily related to oxidation processes of active organic radicals that are dissolved in water or contained in suspended matter, with particles no larger than a few microns in size. The substances that can emit light are produced in the top zone of the lake. The light field at large depth is formed by the transport of luminescent matter to the deep layers through straight sinking of the suspended matter, through turbulent processes and through water flows. The luminescence intensity of an isolated water volume decreases exponentially over time, with a time constant between a few days and tens of days. From the data shown on Fig.1 one can roughly estimate the effective vertical speed of luminescent matter as $10 \mathrm{~m} /$ day. We note the bump of the luminescence at $1100 \mathrm{~m}$. This phenomenon, as well as some events presented in [2] where luminous matter propagates through the neutrino detector from below, is likely caused by the transport of luminescent matter from the shore region along the steeply falling bottom to the site of the detector, followed by upwelling (see also section 4).

As was shown in [3], luminescence is very effective marker of hydrophysical, hydrobiological and hydrochemical processes in Lake Baikal. The neutrino detectors with their net of optical detectors are excellent devices to use the luminescence as instrument for the 
site studies. Since 1993 the neutrino detectors delivered important results about deep water dynamics. The most surprising observations were: the strong heterogeneity and nonstationarity of the water motion, internal waves with amplitudes up to some hundreds meters, and events of vertical water speeds of a few $\mathrm{cm} / \mathrm{sec}$. Some of them are presented in detail in the papers [2] and [4].

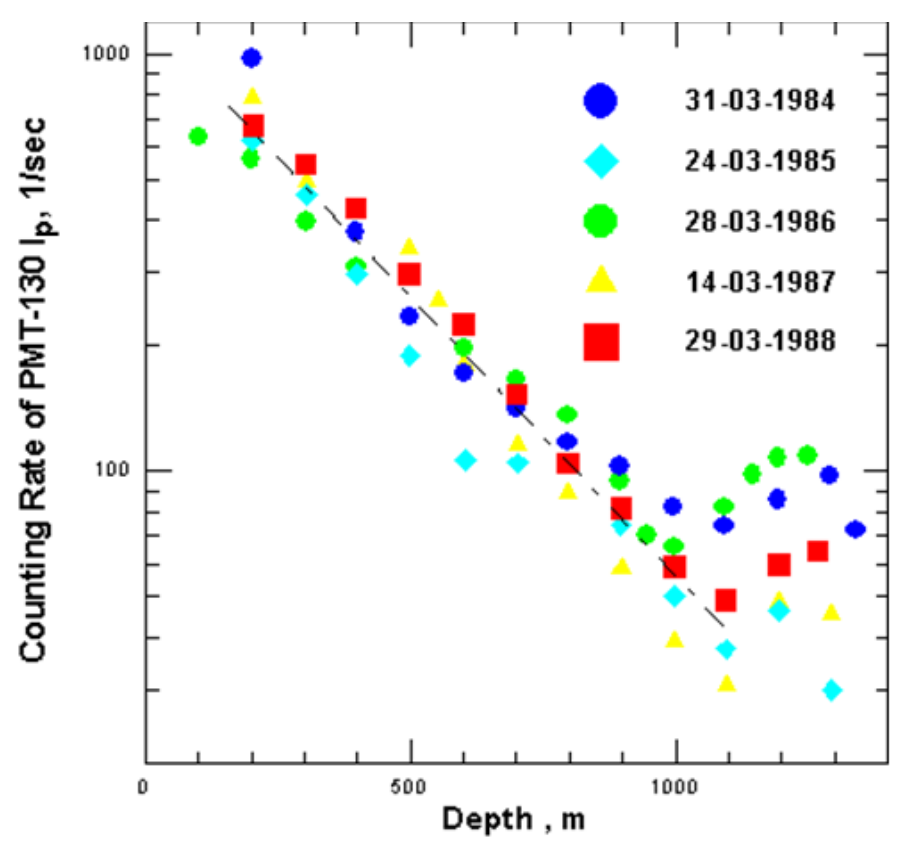

Figure 1. PMT counting rate $I_{p}$ depending on depth. The photon flux can be calculated from the countig rate as $\mathrm{E}_{\mathrm{p}}\left(\right.$ photon $\left.\mathrm{cm}^{-2} \sec ^{-1}\right)=(3,5 \pm 1,0) \mathrm{I}_{\mathrm{p}} \mathrm{sec}^{-1}$

\section{Optical properties of Baikal water}

We have constructed several instruments for long-term in situ monitoring of the inherent optical properties: the absorption length $\mathrm{L}_{\mathrm{a}}(\lambda)$, the scattering length $\mathrm{L}_{b}(\lambda)$, and the scattering phase function $\chi(\theta, \lambda)$, where $\lambda$ is the wavelength and $\theta$ the scattering angle. The main distinctive feature of our instruments is that the distance between light sources and light receivers in situ can be changed over a wide range. That allows to measure the signal amplitude versus distance and to determine the optical properties by a fit, without any calibration procedures. For deep water at the Baikal-GVD site, we measure the following values [6]: absorption length $\mathrm{L}_{\mathrm{a}}(488 \mathrm{~nm})=20-25 \mathrm{~m}$ and $\mathrm{L}_{\mathrm{a}}(400 \mathrm{~nm})=8-12 \mathrm{~m}$, scattering length $\mathrm{L}_{\mathrm{b}}=40-70 \mathrm{~m}$ and - weakly dependent on the wavelength - average cosine of the scattering angle $<\cos (\theta)>=0.9 \pm 0.05$.

\section{Hydrophysical studies}

The water temperature has a substantial impact on the hydrophysical, hydrobiological and hydrochemical processes in the lake, moreover on the depth dependence of the sound speed and the trajectories of acoustic signals. At the same time, the water temperature is a very efficient tracer to study hydro-physical phenomena. We started regular long-term, high precision measurements of the temperature in March 1999, in cooperation with the Swiss Federal Institute of Environmental Science and Technology (EAWAG) and the Limnological Institute of the Siberian Branch of the Russian Academy of Science. We have 
been using more than 50 temperature loggers TR-1000, TR-1050 and other types manufactured by a commercial company. They have been distributed between the lake bottom and $15 \mathrm{~m}$ water depth at four moorings located in the vicinity of Baikal-GVD. All around the year and each 10 minutes, these instruments measure the temperature each with a resolution better than $0,002{ }^{\circ} \mathrm{C}$.

Among the most active hydrophysical phenomena is the mixing of water due to free convection. It leads to homothermy of $250 \mathrm{~m}$ layer of the Lake twice per year. The water temperature below $300 \mathrm{~m}$ slowly decreases with depth, and the density of water is perfectly stratified all around the year. However, a few times per year the positive temperature variations up to $1000 \mathrm{~m}$ depth are observed. The structures of the variations at different depths are correlated, although the amplitude decreases with depth. These disturbances have a maximum magnitude in a period close to the autumn homothermy at the top layer of the lake and are connected with the intrusion of relatively warm water from upper layers. Two basic mechanisms resulting in the water displacement over a large range of depths were suggested: thermal bars [6] and fronts [7]. However, data never showed cases when such vertical disturbances reach down to the bottom. Therefore, they cannot be responsible for the huge cold water intrusions in the near-bottom zone which have been regularly observed around the time of convective surface mixing in June and December/January (Fig. 2 , left) and which have a typical volume of $50-100 \mathrm{~km}^{3}$. Such intrusions play a crucial role in oxygenation of the deepest layers of the lake. They also lead to an efficient recycling of the nutrients from the deep water which is of the same order of magnitude as the external nutrient inputs. To be able to predict changes in the deep water renewal rates in a changing environment, it is essential to understand the underlying mechanism.
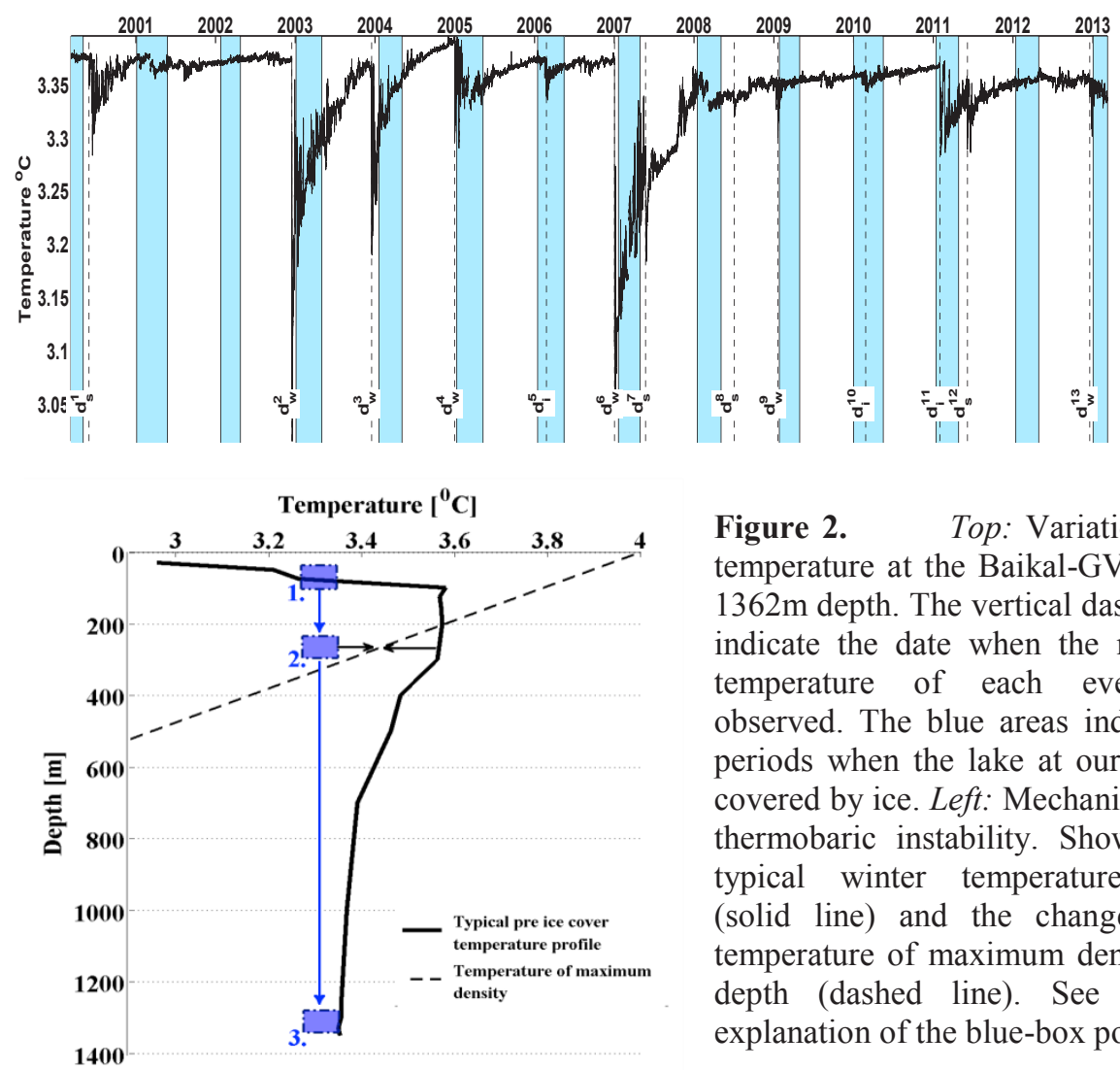

Figure 2. Top: Variation of the temperature at the Baikal-GVD site at $1362 \mathrm{~m}$ depth. The vertical dashed lines indicate the date when the minimum temperature of each event was observed. The blue areas indicate the periods when the lake at our site was covered by ice. Left: Mechanism of the thermobaric instability. Shown are a typical winter temperature profile (solid line) and the change of the temperature of maximum density with depth (dashed line). See text for explanation of the blue-box positions. 
The explanation of this phenomenon is based on a model proposed in [8] and on experimental data taken in the framework the Baikal Neutrino experiment $[9,10]$. The temperature of the maximum density (TMS) of fresh water decreases by $0.2{ }^{\circ} \mathrm{C}$ per $100 \mathrm{~m}$ depth, varying from $4{ }^{\circ} \mathrm{C}$ at the surface to $1,2{ }^{\circ} \mathrm{C}$ at the depth of Baikal-GVD. In December/early January and in June/early July the temperature of surface water is below the TMS across the shallowest $\sim 400$ meters and also slightly colder than the deep water (Fig.2, right). If at this time some packet of near-surface water (position 1 of the blue box in the figure) is forced downwards, it will cross a potential energy barrier at $\sim 300 \mathrm{~m}$ depth (position 2), whereafter its density is higher than that of the ambient water (i.e its distance to the TMS line is smaller than that of the ambient water). Therefore it will freely sink down to the bottom of the lake or to its equilibrium depth (position 3 in the figure).

In $[9,10]$ periods have been observed when a North-East wind with speed $>6 \mathrm{~m} / \mathrm{sec}$ initiated water currents along shore with speed $>25 \mathrm{~cm} / \mathrm{sec}$ at $15 \mathrm{~m}$ depth. The authors could show that, due to the Coriolis force, near-surface water was downwelling along the steep West-North slope of the lake and eventually reached the GVD location. There, the time difference between temperatures observed $3 \mathrm{~m}$ above ground with thermistors at a distance of 1,0 km from shore (547 m depth) and at a distance of 3,2 km from shore (1362 $\mathrm{m}$ depth) showed that cold water packets moved down along the bottom slope with a speed of $1 \mathrm{~km} /$ day. Very likely, this mechanism supplies deep layers of the lake by oxygen and luminescent matter. Later, such events are followed to upwelling mechanism [11].

\section{Geophysical studies}

Using the infrastructure of the Neutrino Observatory a multi-year electromagnetic experiment for studying processes in the hydrosphere and related geospheres is conducted, a central part of which is the monitoring of the vertical component of the natural electric field [12]. The vertical electric field $E_{z}$ in the hydrosphere is not contaminated by the telluric component and therefore can efficiently be used to monitor various processes in the hydrosphere itself, in the lithosphere and in the atmosphere. With this aim, we monitor $E_{z}$ over the baseline of surface to floor since 2003. The absence of a telluric component was confirmed experimentally. The effect and precursors of a close earthquake (see Fig.3), variations of the water transport, and, for the first time, variations of the Global Electric Circuit (GEC) current in the conducting Earth have been studied [12].

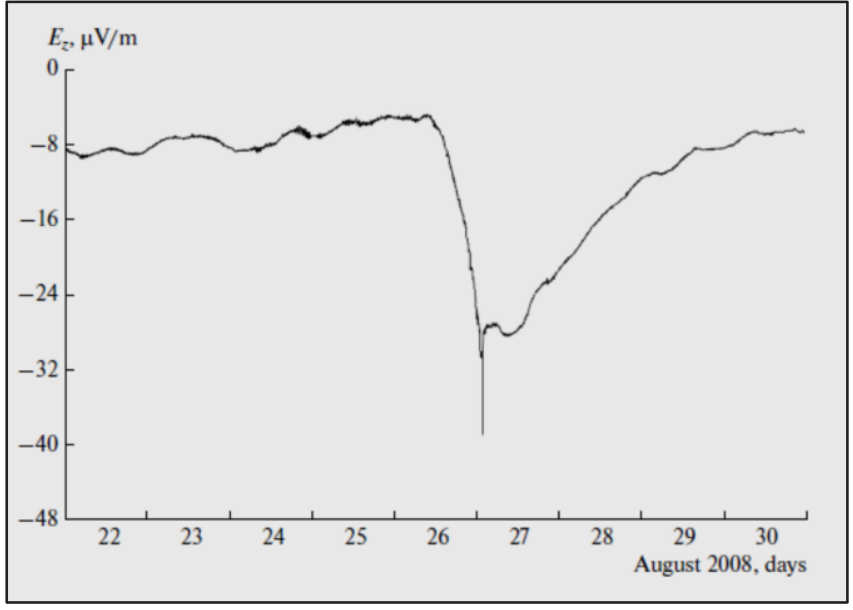

Figure 3. Change in the vertical component of electrical field (minutely averaged data) in August 2008, a few days before after the August 27 earthquake. The moment at which the earthquake occurred corresponds to the spike. 
The lack of a telluric component in $E_{z}$ is confirmed and justified by simulation; we observed a dominant role of the GEC current field over periods from 10 hours to 10 days Within periods of the predominance of the GEC current field, a correlation between $E_{z}$ and the flux of solar X-ray radiation was detected. This correlation is characterized by a large time lag between X-ray and $E_{z}$ variations which is evidence for the complex, mediated mechanism of the solar activity influence on the current [13-14]. Detailed geo-electric models of the Baikal rift have been worked out, by which competing hypotheses on its deep structure can be compared. On this basis, the calculations of the field and electrical conductivity functions in the region of the experiment have been performed [12].

\section{References}

1. Vladimir Aynutdinov et al., these proceedings.

2. Rastislav Dvornicky et al., these proceedings

3. L.B.Bezrukov et al, Izvestia Atmospheric and Oceanic Physics 34, 85 (1998).

4. I.A.Belolaptikov et al, Izvestia Atmospheric and Oceanic Physics 34, 78 (1998).

5. A. Avrorin et al, NIM A 693, 186 (2012).

6. M.N. Shimaraev, N.G. Granin, A.A. Zhdanov, Limnol. Oceanogr. 38, 1068 (1993)

7. P.P. Sherstyankin, Dokl. RAN 326, 366 (1992)

8. Weiss, R.F., E.C. Carmack, and V.M. Koropalov, Nature, 349, 665 (1991)

9. M. Schmid, N. Budnev, N. Granin, M. Sturm Geophysical Research Letters 35, L09605 (2008).

10. C. Tsimitri et al, Journal of Geophysical Research: Oceans 120, 1508 (2015).

11. E.Troitskaya et al. Aquatic Science. 77, 171 (2015).

12. S.M. Korotaev et al, Izv., Atmospheric and Oceanic Phys. 54, 1569 (2018)

13. S.M. Korotaev et al, Geomagn. Aeron. 53, 769 (2013).

14. S.M Korotaev et al, Geomagn. Aeron. 58, 142 (2018). 